\title{
Rasgos morfológicos alrededor de la Isla del Coco y de sus montes submarinos vecinos, Pacífico de Costa Rica
}

Omar G. Lizano 1,2,3

1. Centro de Investigaciones Geofísicas (CIGEFI), Universidad de Costa Rica, 11501-2060 San José, Costa Rica; omar.lizano@ucr.ac.cr

2. Centro de Investigación en Ciencias del Mar y Limnología (CIMAR), Universidad de Costa Rica, 11501-2060 San José, Costa Rica.

3. Departamento de Física Atmosférica, Oceánica y Planetaria (DFAOP), Escuela de Física, Universidad de Costa Rica, 11501-2060. San José, Costa Rica.

Recibido 28-V-2012. Corregido 19-VII-2012. Aceptado 24-IX-2012.

\begin{abstract}
Morphological features around Isla del Coco and its adjacent seamounts, Pacific Costa Rica. The UNESCO GEBCO-08 bathymetric data was used to study the marine provinces around Isla del Coco. Seamounts with depths of less than $1000 \mathrm{~m}$ from the sea surface were analyzed. Seamounts southward, southeastward and westward of the island, were identified at depths of $300 \mathrm{~m}$ from the sea surface, and with heights up to $2700 \mathrm{~m}$ from the marine bottom. The ecological interconnection of these mountains with Isla del Coco has to be effective, as it could also be within the biological corridor of Eastern Tropical Pacific. A better resolution study around Isla del Coco was done to identify details of the insular platform. A seamount to $14.8 \mathrm{~km}$ northeast of the island was found, with measured depths of $30-40 \mathrm{~m}$, and so far, not reported. This feature explains the presence of fishermen around this site, which is known by them as a place with high productivity. Although more studies are needed, the limits and environmental policies should be modified so as to adequately protect these seamounts, which probably have an important ecological connection with Isla del Coco. Citation: Lizano, O.G. 2012. Rasgos morfológicos alrededor de la Isla del Coco y de sus montes submarinos vecinos, Pacífico de Costa Rica. Rev. Biol. Trop. 60 (Suppl. 3): 43-51. Epub 2012 Dec 01.
\end{abstract}

Key words: bathymetry, insular shelf, Cocos Island, Costa Rica, seamounts.

El artículo sobre la batimetría de los alrededores de la Isla del Coco, muestra datos de sondeos e interpolaciones desde la única carta náutica existente para la isla y cuya profundidad no va más allá de los 183m (100 brazadas) (Lizano 2001). Con la pobre resolución espacial de 5 grados de los datos públicos que habían hasta ese momento, la Figura 1 de dicho artículo, muestra la posición de la isla en la Cordillera Volcánica del Coco (CVC) (Rojas \& Alvarado 2012). Datos más recientes (0.5 grados) de GEBCO_08 (The General Bathymetric Chart of the Oceans) de la Intergovernmental Oceanographic Commission (IOC), distingue más claramente la posición de la isla en la CVC (Lizano 2008). Además, del valor biológico indiscutible de la Isla del Coco (Cortés 2007, 2008, Montoya 2007), declarada Patrimonio de la Humanidad en 1997 (Bolívar et al. 2000, Montoya 2007), ha habido un gran interés por conocer los alrededores de la Isla del Coco y sus posibles interconexiones ecológicas con los montes submarinos vecinos o el corredor marino (Meneses 2005, Rodríguez 2005, Rothschild 2005), como lo expresa también, la página de la Fundación Amigos de la Isla del Coco (http:// www.cocosisland.org/corredor-marino.html). Según Rojas \& Alvarado (2012), la isla y los montes submarinos, son el resultado de anomalías térmicas que surgieron desde el manto a través las fisuras de la corteza oceánica y que tienen una edad de entre 2.2 y $1.5 \mathrm{Ma}$ 


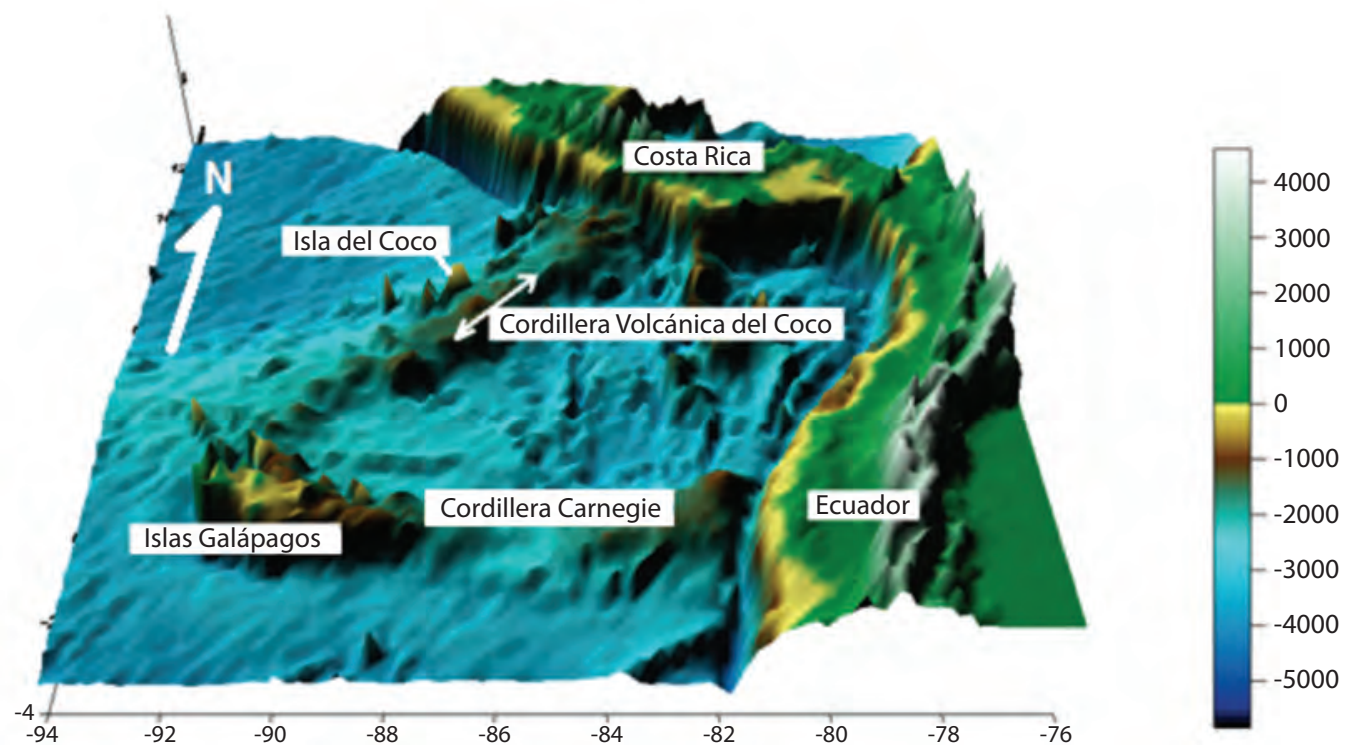

Fig. 1. Batimetría de Pacífico Tropical Este en tres dimensiones.

(millones de años). Similar estimación propone O'Connor et al. (2007), quien además indica que estos rasgos submarinos son más jóvenes que las Islas Galápagos que emergieron aproximadamente hace $15 \mathrm{Ma}$ (Rojas \& Alvarado 2012). El estudio de los montes submarinos es importante ya que son sitios que favorecen la productividad, la biomasa y la diversidad. Son verdaderos oasis biológicos que contribuyen con la ecología marina (Lavelle \& Mohn 2010). Desde el punto de vista físico, los montes submarinos actúan como columnas que modifican el flujo, donde la energía de las mareas puede generar ondas internas (Garrett 2003), turbulencia y remolinos (Roger 1978). Por otro lado, se ha probado que con la presencia de montes se generan circulaciones que atrapan partículas y organismos, lo cual está relacionado con alta biodiversidad que pueden sustentar (Lavelle \& Mohn 2010).

En este trabajo se hace uso de los datos nuevos de resolución espacial de $30 \mathrm{seg}(925.5 \mathrm{~m})$ de GEBCO, que permiten identificar los rasgos batimétricos de la plataforma submarina de la isla. Esta morfología no se conocía, y aunque las cartas náuticas existentes tienen alguna información batimétrica de los montes submarinos vecinos, no se conocía con el detalle que muestra este estudio. La información suministrada permitirá guiar nuevas investigaciones y elaborar políticas de protección ambiental a los montes submarinos, en especial al monte submarino más próximo de la isla, también desconocido, que está a solo $14.5 \mathrm{~km}$ de Manuelita, y que está sometido a una alta explotación del recurso del pesquero.

\section{MATERIALES Y MÉTODOS}

Los datos de GEBCO (Becker et al. 2009): "The GEBCO_08 Grid", ver. 20091120 (http:// www.gebco.net) con resolución espacial de 30 segundos fueron extraídos con el paquete Grid Viewer ver. 12.13 de GEBCO de la región delimitada por $4^{\circ} \mathrm{S}-12^{\circ} \mathrm{N}$ y $94^{\circ}-78^{\circ} \mathrm{W}$. Esta región y otras subregiones fueron extraídas y graficadas con el programa SURFER ver. 9. Contornos topográficos de la Isla del Coco fueron digitalizados de la hoja topográfica del Instituto Geográfico Nacional (IGN). Dado que las posiciones de la carta náutica de la Isla del Coco (DMA No. 21 62, 10 ${ }^{\mathrm{ma}}$ edn., septiembre 
8, 1984), está desplazada unos $350 \mathrm{~m}$ de las posiciones del GPS (mediciones de este autor), se desplazó la carta náutica al mapa IGN con paquete ARGIS ver. 10. El programa SURFER ver. 9.0 fue utilizado para editar y graficar todos los datos mostrados en este artículo. Una ecosonda Garmin 520S del MV Sea Hunter del Undersea Hunter Group, fue utilizada para hacer mediciones comprobatorias del monte submarino al noreste de la Isla del Coco.

\section{RESULTADOS}

Los rasgos batimétricos del Pacífico Tropical Este muestran las dos principales cordilleras (Fig. 1): la Cordillera Volcánica del Coco, extendiéndose en un eje noreste-sureste, y la Cordillera Carnegie, que se extiende al este. O'Connors et al. (2007), sugiere que estas mayores crestas asísmicas son trayectorias gemelas de puntos calientes de la placas de Nazca y Coco creadas en el punto caliente de Las Galápagos. En la figura se puede identificar que la posición de la Isla del Coco no está en el eje central de la cordillera, sino sobre el flanco oeste de la misma.
Sobre ese mismo flanco hay al menos 3 montes submarinos con alturas de más de $2000 \mathrm{~m}$ y cuyas cumbres tienen profundidades de menos de 1000m (Fig. 2 y Cuadro 1). Estos montes están dentro de la así llamada "provincia" de la Isla del Coco (O’Connors et al. 2007).

La Isla del Coco es una montaña que se levanta desde el piso oceánico hasta la cumbre del Cerro Iglesias, unos 2574m (Cuadro 1), según los datos del GEBOCO_08. La estructura morfotectónica propiamente sobre la cual se levanta la Isla del Coco, era desconocida, pues la única hoja batimétrica existente (Lizano 2001), no alcanzaba para visualizar la isla totalmente. Esto se puede identificar desde la Figura 2, donde el cuadro interno del recuadro 1 , corresponde al área que abarca dicha batimetría. La batimetría digitalizada de esta hoja, donde además se ha agregado la topografía, se presenta en la Figura 3. Se nota que alrededor de la isla hay una plataforma insular con profundidades poco variables hasta la profundidad de unos $100 \mathrm{~m}$, a partir de la cual cambia drásticamente la pendiente de esa plataforma. Sobre el borde noroeste, y al frente de Bahía Wafer,

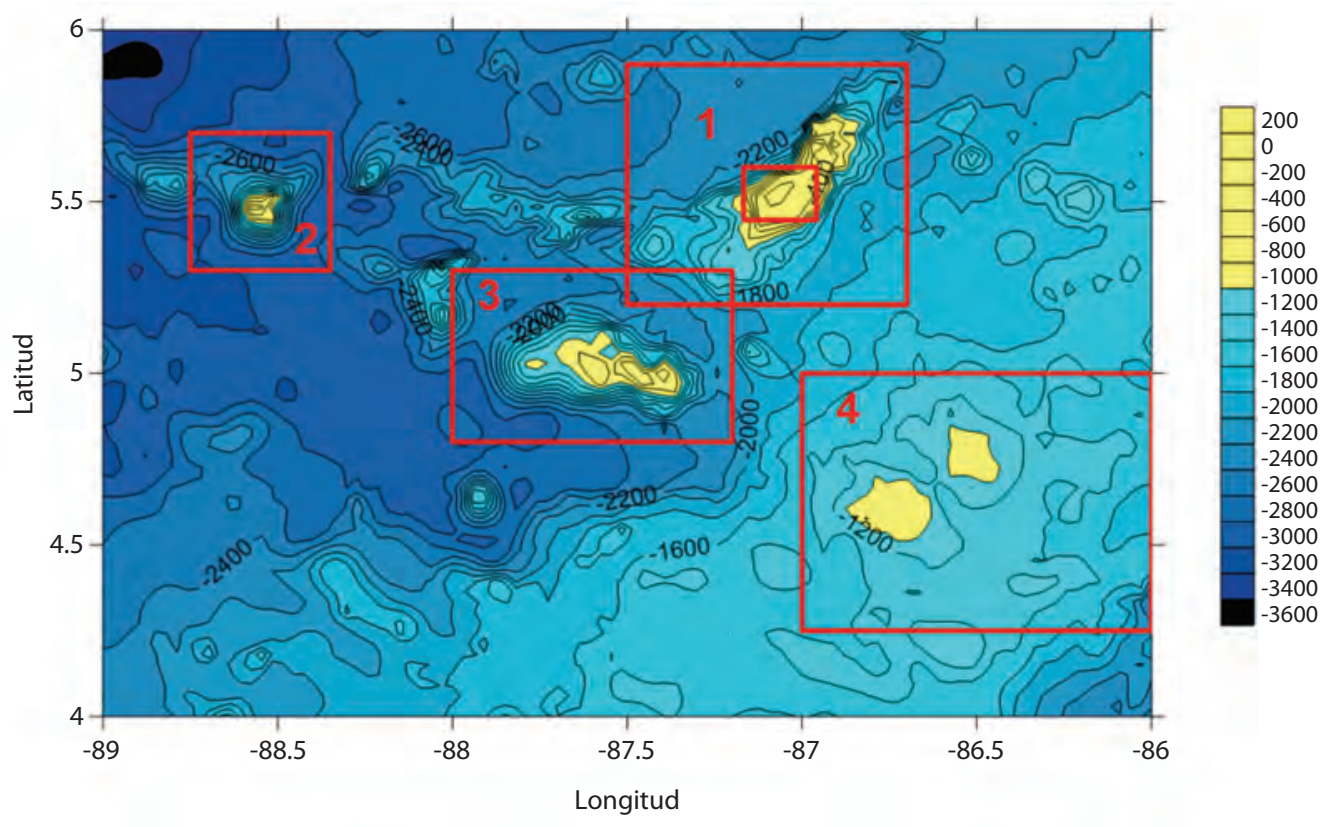

Fig. 2. Contornos batimétricos de alrededor de la Isla del Coco con montañas submarinas menores de $1000 \mathrm{~m}$. 


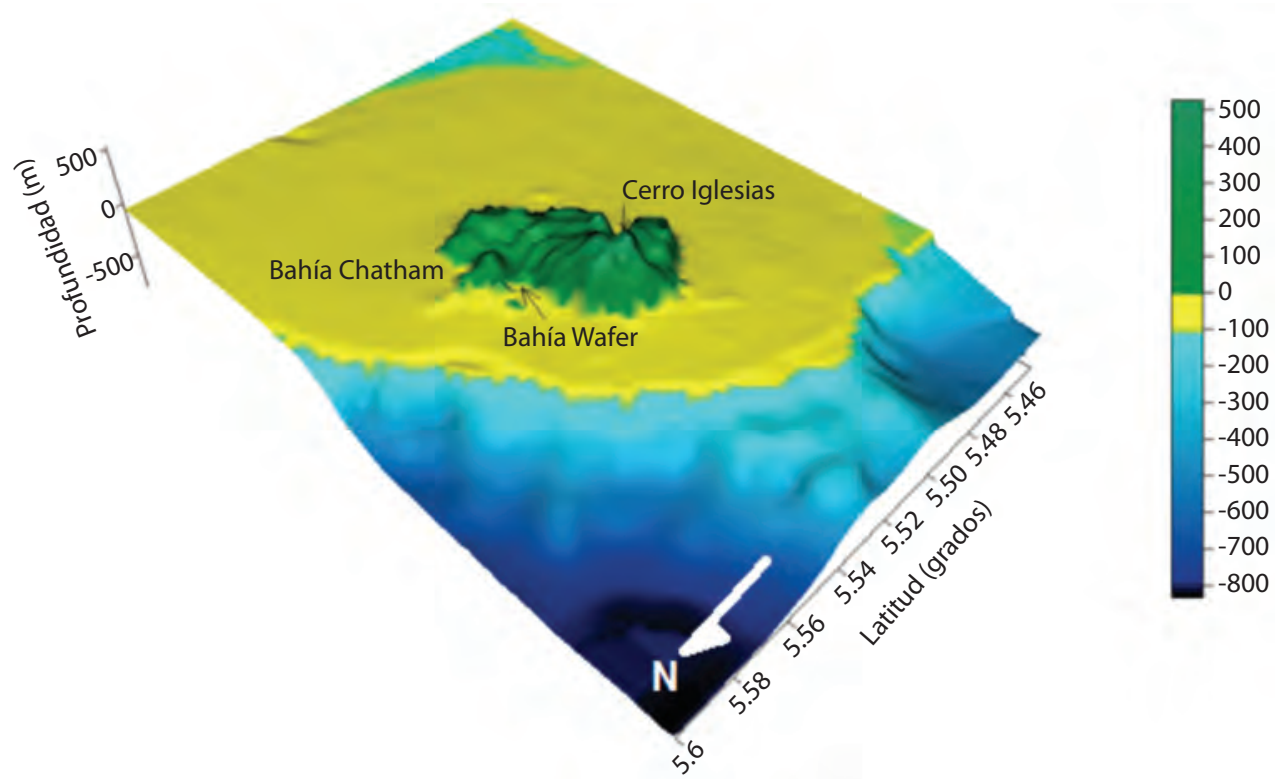

Fig. 3. Plataforma insular y la topografía en tres dimensiones de la Isla del Coco vista desde el noroeste.

\section{CUADRO 1}

Alturas de los montes submarinos vecinos de la Isla del Coco según los datos GEBCO_08.

\begin{tabular}{clccccc} 
No. & \multicolumn{1}{c}{ Monte } & Lat/Long & Pbase & Pcumb. & Alt. monte & Dist/Dir I. Coco \\
1 & I. Coco & $5.5220^{\circ} / 87.0758^{\circ}$ & 2440 & +107 & 2574 & \\
2 & Cortés & $4.4690^{\circ} / 86.6201^{\circ}$ & 3040 & -302 & 2738 & $165 / 266^{\circ}$ \\
3 & Las Gemelas & $5.0115^{\circ} / 87.5835^{\circ}$ & 2900 & -480 & 2420 & $72 / 211^{\circ}$ \\
4 & Sin nombre & $5.4899^{\circ} / 88.5557^{\circ}$ & 1700 & -860 & 840 & $109 / 164^{\circ}$ \\
\hline
\end{tabular}

Pbase: profundidad en metros desde la superficie del mar hasta la base más profunda del monte, según los recuadros de Fig. 2.

Pcumb.: profundidad en metros desde la superficie del mar a la altura máxima del monte.

Alt. Monte: altura en metros del monte desde la base.

Dist/Dir ICoco: Distancia en kilómetros, dirección en grados respecto al norte verdadero, medidos desde Bahía Chatham, Isla del Coco.

el talud insular cambia drásticamente a partir de los $100 \mathrm{~m}$ en una distancia de $2.5-3.0 \mathrm{~km}$ de la costa (Fig. 3). El borde este de la plataforma insular es más extenso $(8-10 \mathrm{~km})$, pero no se puede identificar su alcance desde estos datos. Según Rojas \& Alvarado (2012), esta plataforma insular plana, es probablemente el resultado de la erosión marina en los últimos dos máximos glaciales.

Los datos recientes de GEBCO_08 permiten visualizar completamente el entorno morfológico de la Isla del Coco. La región del recuadro 1 de la Figura 2, sobre las proximidades de la Isla del Coco, se muestra en detalle en las Figuras 4 y 5.

La Isla del Coco surge sobre el costado suroeste de este promontorio oceánico que muestra las Figuras 4 o 5, que se extiende de suroeste a noreste y que se levanta más de $2500 \mathrm{~m}$ sobre el piso oceánico. También se identifica el alcance de la plataforma insular que es más ancha al este de la isla, y que no 


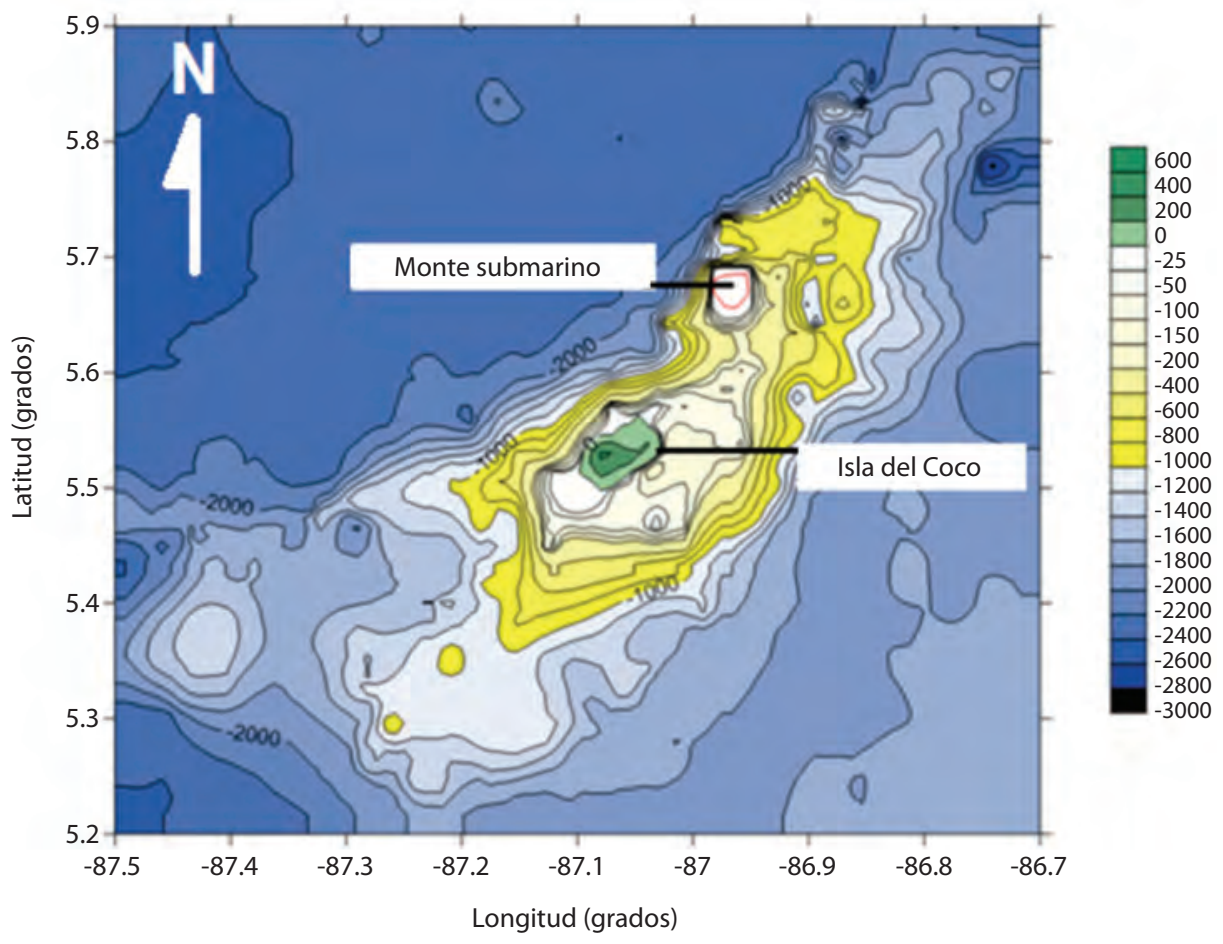

Fig. 4. Contornos de profundidad en metros alrededor de la Isla del Coco.

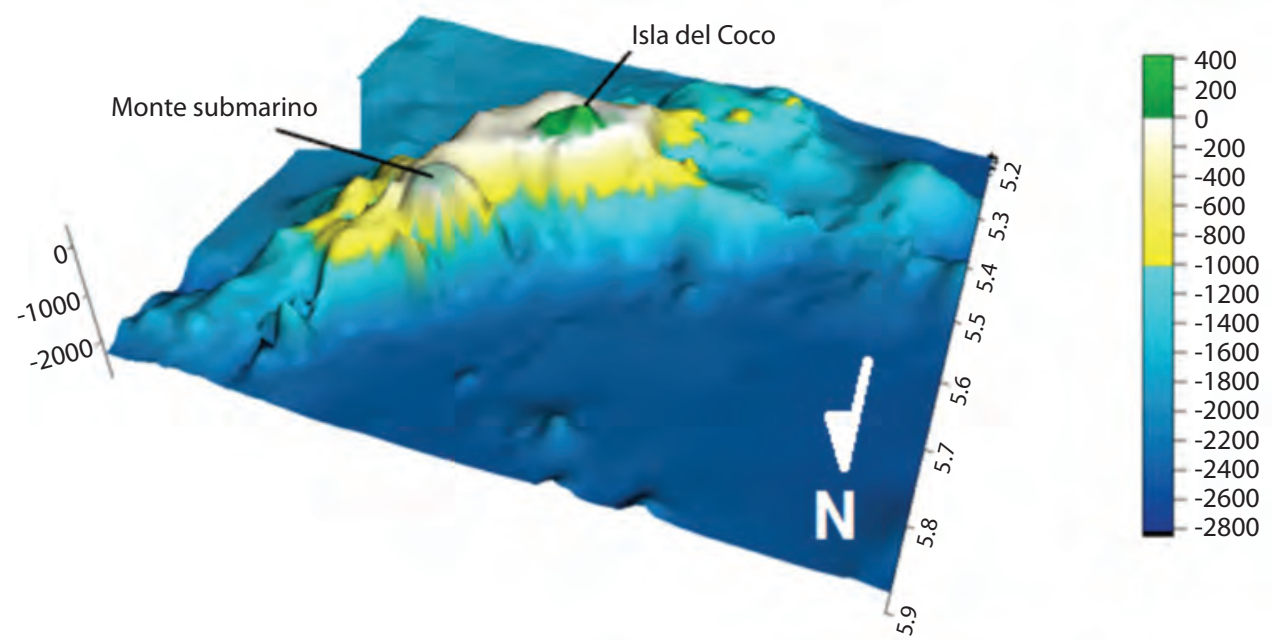

Fig. 5. Modelo de Elevación Digital (MED) de la Fig. 4, visto desde el noroeste.

había podido definirse desde la carta náutica que se tenía.

Un rasgo importante en esta plataforma es el monte submarino que está al noreste de la isla (Figs. 4, 5). Este monte es un pico achatado (según los datos del GEBCO_08), de forma cuadrada con longitud aproximada de $3700 \mathrm{~m}$. Está a $14.8 \mathrm{~km}$ de Isla Manuelita, con dirección 
$39^{\circ} \mathrm{N}$, y situado sobre ese mismo dorsal de la isla, pero separado por una depresión batimétrica cuya máxima profundidad es $180 \mathrm{~m}$ (Figs. $4,5)$. La pared oeste de este monte tiene pendientes muy fuertes, son paredes prácticamente verticales, muy parecidas a las formaciones que se notan en la isla propiamente. En menos de $60-70 \mathrm{~m}$, se cambia de profundidades de $2 \mathrm{~m}$ (profundidad del monte según los datos GEBCO_08), a 2000m (Figs. 4, 5). Mediciones que se hicieron en la Expedición UCR-UNACOCO-V del 13 al 23 de marzo del 2012, registraron sondeos alrededor de monte submarino de entre 35 y $40 \mathrm{~m}$ de profundidad con la ecosonda del MV Sea Hunter, valores que se repitieron pasando cuatro veces por las coordenadas que ubican a este monte. Aún no se conoce nombre de este rasgo morfológico de la isla. Las diferencias en general entre las mediciones y los valores suministradas por GEBCO_08, se deben a que estos últimos datos son una interpolación de datos de barcos, o de anomalías de gravedad de satélites, cuyos cálculos pueden no ser muy exactos, ya que dependen del tipo de instrumento utilizado para medir profundidad y de la densidad de valores espaciales existentes en el lugar (Becker et al. 2009).
En la Fig. 2 se identifican otros montes submarinos alrededor de la Isla del Coco (recuadros 2, 3 y 4). El que está al oeste (recuadro 2 en Fig. 2), es más alto que la Isla del Coco, pero está en una región más profunda, como muestra la Figura 2. Este monte es un cono bien formado en cuya cumbre surgen varios picos. Propongo como nombre de este monte: Monte Submarino Cortés (Fig. 6), en honor al trabajo de años en estas regiones, del investigador del CIMAR, Dr. Jorge Cortés Nuñez.

El monte submarino que aparece en el recuadro 3 de la Figura 2, es llamado por los pescadores "Los Picos de la Isla del Coco", y en el análisis de vacíos de conservación GRUAS II, se les ha llamado Monte Submarino Las Gemelas (SINAC 2009). Recientemente fueron visitados con un sumergible y se han empezado a estudiar (Cortés et al.2012, Starr et al.2012). Es una serranía bastante irregular, con varios picos en su cima (Fig. 7). El pico que se identifica sobre el costado norte tiene una altura de aproximadamente $600 \mathrm{~m}$.

Los montes submarinos en el recuadro 4 de Fig. 2, están sobre la Cordillera Volcánica del Coco, y tienen una base menos profunda que los anteriores, mas sin embargo,

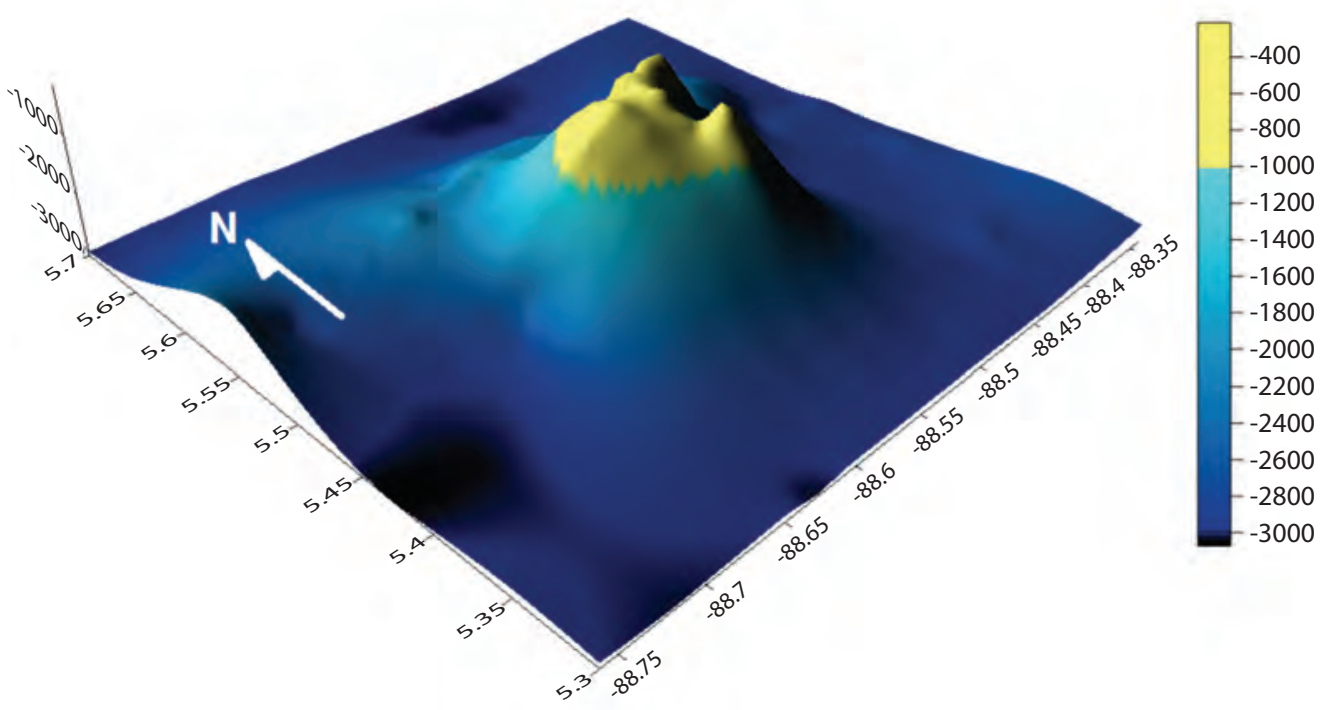

Fig. 6. Monte Submarino Cortés. 


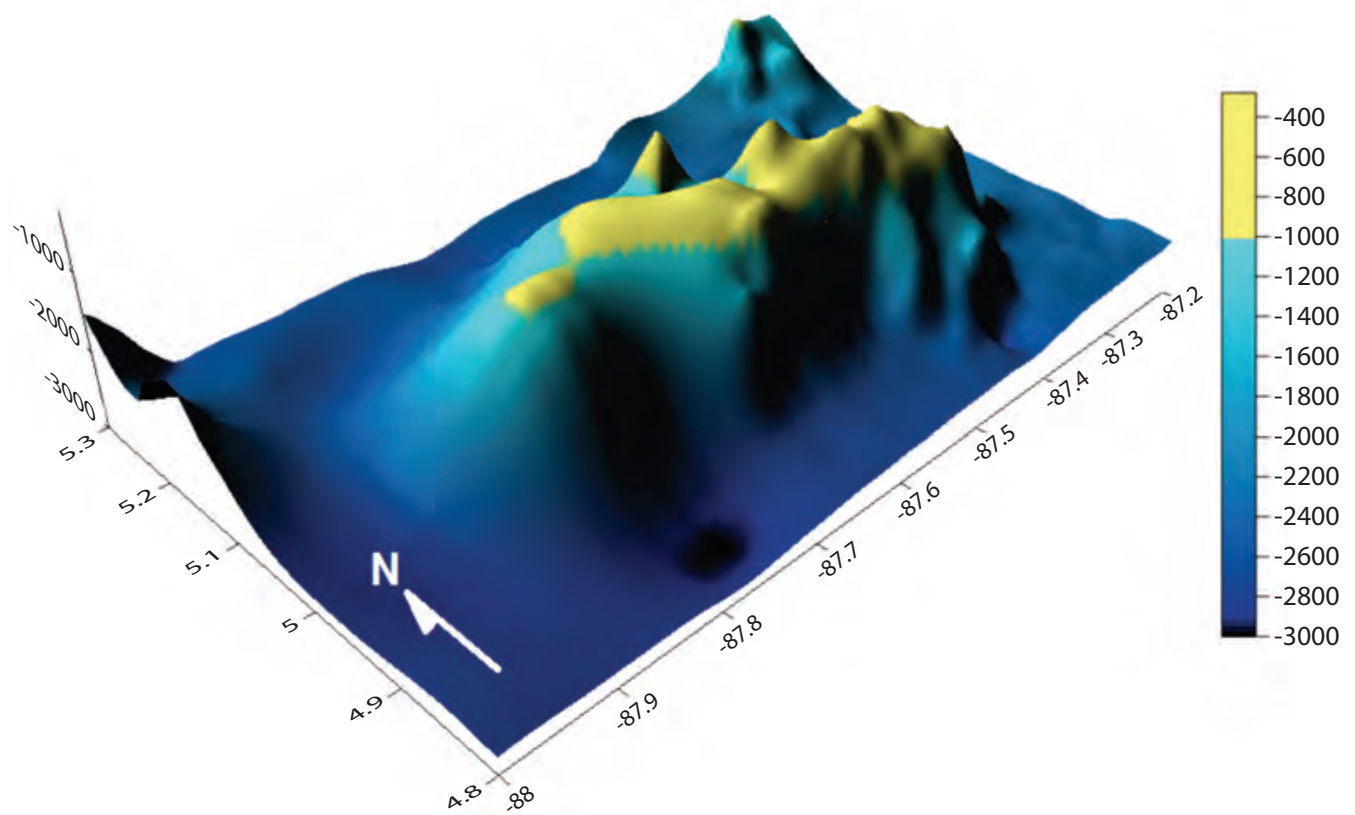

Fig. 7. Montes submarinos Las Gemelas.

están a una profundidad mayor respecto de la superficie del mar (aproximadamente $800 \mathrm{~m}$, Cuadro 1). Son más extensos y achatados que los anteriores montes.

\section{DISCUSIÓN}

Los datos recientes de GEBCO_08, permitieron detallar aquellos montes submarinos más cercanos a la Isla del Coco cuyas profundidades son menores de $1000 \mathrm{~m}$, y que probablemente tienen una interconexión ecológica (Starr et al. 2012). A su vez, estos montes submarinos son parte del "Corredor Marino de Conservación del Pacifico Este Tropical" (Meneses 2005, Rodríguez 2005, Rothschild 2005). Esta es la región geográficamente compuesta por cinco islas/archipiélagos en el Pacífico Tropical Este de Ecuador (Galápagos), Colombia (Gorgona y Malpelo), Panamá (Coiba) y Costa Rica (Coco). Se argumenta que estas áreas tienen condiciones biogeográficas y geológicas similares, con intercambios genéticos y presencia de varias especies marinas y terrestres con un origen común (Anónimo 2004).
Los dos montes, uno al sureste, y el otro al suroeste (Fig. 2), tienen menos de $500 \mathrm{~m}$ de profundidad. Cortés y Blum (2008) describen la alta diversidad de estos ambientes de aguas más profundas en la Isla del Coco. Las observaciones que se han hecho con sumergibles del monte submarino Las Gemelas, muestran importante diversidad marina en sus cumbres (Starr et al. 2012, J. Cortés, comun. pers.). La relación ecológica que tengan estos montes con la isla, son un tema de investigación necesaria (Cortés \& Blum 2008).

Otro de los objetivos de esta investigación fue detallar completamente la estructura morfotectónica sobre la cual se levanta la Isla del Coco. Un resultado sorprendente fue descubrir un rasgo batimétrico hasta ahora desconocido, el monte submarino a $14.8 \mathrm{Km}$ al noreste de la isla, y a una profundidad de tan solo $30-40 \mathrm{~m}$. Se necesitan más observaciones y mediciones con un equipo de sondeo de mayor confiabilidad o con submergible que evidencie su estado ambiental. La existencia de este monte submarino cerca de la Isla del Coco cambia la concepción biológica, política y ambiental que 
se tiene sobre la isla y su entorno. Los límites de protección de la isla se habían ampliado hasta $15 \mathrm{~km}$ (Cajiao 2008). Dado que el monte submarino está dentro del borde de ese círculo imaginario, deberán evaluarse las políticas vigentes de protección de los recursos marinos a la luz de esta nueva información, de manera que se protejan apropiadamente, como ya lo había sugerido Quesada (2006). Por otro lado, la existencia de este monte explica la gran productividad de este sitio, y la presencia de la gran cantidad de pescadores que siempre se encuentra alrededor (Guardarecursos del Parque Nacional Isla del Coco, comun. pers.). Es muy probable que con las prácticas de arrastre que usualmente utilizan los pescadores, o hasta las mismas redes que pudieran estar en el sitio (que está dentro del Parque Nacional Isla del Coco), habrían destruido por años cualquier estructura marina alrededor del mismo, un tema de estudio inmediato que surge de esta contribución.

\section{AGRADECIMIENTOS}

Al Capitán del Sea Hunter, Raúl Méndez Venegas, por la paciencia de pasar una y otra vez para comprobar la existencia del monte submarino al noreste de la Isla del Coco. A Jorge Cortés por confiar y apoyar la investigación particular alrededor de este monte. A CONARE por el apoyo económico al proyecto "Interacciones océano-atmósfera y la biodiversidad marina de la Isla del Coco, Costa Rica" dentro del Programa "Investigaciones marinas, limnológicas y atmosféricas en el Parque Nacional Isla del Coco (IMLAIC)", al CIMAR y la Vicerrectoría de Investigación.

\section{RESUMEN}

Se utilizaron los datos batimétricos GEBCO-08 de la UNESCO para estudiar las provincias marinas alrededor de la Isla del Coco. Se analizaron las montañas submarinas que tuvieran menos de $1000 \mathrm{~m}$ de profundidad en esta cordillera. Montes submarinos localizados al sur, sureste y oeste de la isla, fueron identificados con profundidades de $300 \mathrm{~m}$ desde la superficie del mar, y con alturas de hasta $2700 \mathrm{~m}$ desde el fondo marino. La interconexión ecológica con la
Isla del Coco de estos montes debe ser efectiva, como lo serán también dentro del corredor biológico del Pacifico Tropical Este. Se realizó un estudio particular de mayor resolución alrededor de la Isla del Coco para identificar los alcances de la plataforma de la isla. Se encontró un monte submarino a $14.8 \mathrm{~km}$ al noreste de la isla, con profundidades medidas de 30-40m, que no había sido reportado. Con este rasgo submarino se explica la presencia de pescadores alrededor del este sitio, un sitio conocido en el ambiente de los pescadores, pero que aún no se había asociado a algún fenómeno oceánico en particular. Aunque más estudio es necesario, de comprobarse la conectividad biológica entre los montes submarinos y la Isla del Coco, los límites y las políticas ambientales deberán ser modificados, de manera que se proteja adecuadamente este sistema ecológico.

Palabras clave: Batimetría, plataforma insular, Isla del Coco, Costa Rica, montañas submarinas.

\section{REFERENCIAS}

Anónimo. 2004. Corredor Marino de Conservación del Pácífico Este Tropical, entre las islas Coco-Galápagos-Malpelo-Coiba-Gorgona. Antecedentes y consideraciones técnicas para su definición. Reunión Regional Ministerial, Hotel TRYP, COROBICI, San José, Costa Rica. 34 p.

Becker, J.J., D.T. Sandwell, W.H.F. Smith, J. Braund, B. Bionder. J. Depner. D. Fabre, J. Factor, S. Ingalls, S-H. Kim, R. Ladner, K. Marks, S. Nelson, A. Pharoah. R. Trimer, J. Von Rosenberg, G. Wallace \& P. Weatherall. 2009. Global bathymetry and elevation data at 30 arc seconds resolution: SRTM30_PLUS. Mar. Geodesy. 32: 355-371.

Bolívar, A., Y. Rovinski \& E. Wo Ching. 2000. La pesca en la Isla del Coco. Estudio integral para el mejoramiento del control pesquero en la zona de influencia del Área de Conservación Marina y Terrestre Isla del Coco. Informe a la Fundación de Amigos de la Isla del Coco, San José, Costa Rica. 101 p.

Cajiao, M. 2008. Aspectos legales relacionados con el Parque Nacional Isla del Coco, Costa Rica. Rev. Biol. Trop. 56 (Supl. 2): 207-214.

Cortés, J. 2007. Coastal morphology and coral reefs. In J. Bundschuh\& G.E. Alvarado (eds.). Central America: Geology, Resources and Hazards. Taylor \& Francis, Londres 1: 185-200.

Cortés, J. 2008. Historia de la investigación marina de la Isla del Coco, Costa Rica. Rev. Biol. Trop. 56 (Supl. 2): $1-18$.

Cortés, J. \& S. Blum. 2008. Life to $450 \mathrm{~m}$ depth at Isla del Coco, Costa Rica. Rev. Biol. Trop. 56 (Suppl. 2): 189-206.

Cortés, J., A. Sánchez-Jiménez, A. Rodríguez-Arrieta, G. Quirós-Barrantes, P.C. González \& S. Blum. 2012. 
Elasmobranchs observed in deep waters (45-330m) at Isla del Coco National Park, Costa Rica (Eastern Tropical Pacific). Rev. Biol. Trop. 60 (Supl. 3): 257-273.

Garret, C. 2003. Internal tides and ocean mixing. Science 301: 1858-1859.

Labelle, W. \& C. Mohn. 2010. Motion, commotion, and biophysical connections at deep ocean seamounts. Oceanography 23: 91-103.

Lizano, O.G. 2001. Batimetría de la plataforma insular alrededor de la Isla del Coco, Costa Rica, Centro América. Rev. Biol. Trop. 49 (Supl. 2): 163-170.

Lizano, O.G. 2008.Dinámica de las aguas alrededor de la Isla del Coco, Costa Rica. Rev. Biol. Trop. 56 (Supl. 2): 31-48.

Meneses, R. 2005. Antecedentes, principios y objetivos del Corredor Marino. Ambientico 138: 4-5.

Montoya, M. 2007. Conozca la Isla del Coco. Una guía para su visitación, P. 35-76. In: Organización de Estudios Tropicales (OET) (eds.). BioCursos para amantes de la Naturaleza. Conozca el Parque Nacional Isla del Coco. La Isla del Tesoro. San José, Costa Rica.

O’Connor, J.M., P. Stoffers, J.R. Wijbrans, T.J. Worthington. 2007. Migration of widespread long-lived volcanism across the Galápagos Volcanic Province: Evidence for a broad hotspot melting anomaly? Earth Planet. Sci. Lett. 263: 339-354.
Quesada, M.A. 2006.CAPITULO XV: Islas: p 195-199. In: V. Nielsen-Muñoz y M.A. Quesada-Alpízar (Eds.). Ambientes Marino Costeros de Costa Rica. Comisión Interdisciplinaria Marino Costera de la Zona Económica Exclusiva de Costa Rica, Informe Técnico. CIMAR, CI, TNC, San José, Costa Rica.

Rodríguez, J. 2005. Historia y carácter del Corredor Marino. Ambientico 138: 6-9.

Roger, A.D. 1978. Ocean eddies generated by seamounts in the North Pacific. Science 199: 1063-1064.

Rojas, W. \& G.E. Alvarado. 2012. Geología y contexto geotectónico de la Isla del Coco y la zona marítima frente al Pacífico central de Costa Rica. Rev. Biol. Trop. 60 (Supl. 3): 15-32.

Rothschild, M. 2005. Corredor Marino: responsabilidad compartida. Ambientico 138: 10-14.

SINAC (Sistema Nacional de Áreas de Conservación). 2009. GRUAS II: Propuesta de ordenamiento territorial para la conservación de la biodiversidad en Costa Rica. Vol. IIII. Análisis de vacíos en la representatividad e integridad de la biodiversidad de los sistemas marinos y costeros. MINAET y TNC, San José, Costa Rica.

Starr, R.M., K. Green \& E. Sala. 2012. Deep-water fish assemblages at Isla del Coco National Park and Las Gemelas Seamounts, Costa Rica. Rev. Biol. Trop. 60 (Supl. 3): 347-362. 
